\title{
Low Complexity Metrics for BICM SISO and MIMO systems
}

\author{
Rizwan Ghaffar, Raymond Knopp \\ Eurecom, 2229 route des Crêtes B.P.193 \\ 06904 Sophia Antipolis Cedex FRANCE \\ Email: rizwan.ghaffar@eurecom.fr, raymond.knopp@eurecom.fr
}

\begin{abstract}
Bit interleaved coded modulation (BICM) because of its improved diversity over fast fading channels is an attractive transmission scheme for future wireless systems. For coded BICM systems, receivers need to employ max log MAP demodulators (demappers) that calculate soft-decision metrics i.e. log-likelihood ratios (LLRs) for the decoder. The complexity of the calculation of these LLRs is exponential in the number of bits per symbol and moreover for systems exploiting spatial dimension (MIMO), the complexity further increases exponentially in the number of transmit antennas.

In this paper we propose matched filter (MF) based low complexity max log MAP bit metrics for BICM single input single output (SISO) and low dimensional BICM MIMO systems using Gray encoded $M$-ary quadrature amplitude modulation (QAM) alphabets. For SISO systems, the maximum likelihood (ML) detector needs computation and comparison of minimum distances between the received symbol and $M$ constellation points on the complex plane for the calculation of each LLR. In this paper we show that these LLRs can be computed precisely from the MF output and therefore do not necessitate any minimum distance calculations. For low dimensional BICM MIMO systems, we further propose a MF based bit metric which successfully trims down one complex dimension of the system thereby reducing complexity. Both these metrics substantially reduce the number of calculations needed for each LLR without compromising the performance and MF being an integral part of all receiver structures facilitates their hardware implementation. Simulation results over Rayleigh fading channels verify similar performance of the simplified metrics as those of the original metrics but with a significant reduction in the complexity.
\end{abstract}

\section{INTRODUCTION}

Future wireless communication systems need robust coding schemes and an appropriate solution in todays wireless world is bit interleaved coded modulation (BICM). Zehavi [1] and Caire [2] realized that the code diversity and therefore the reliability of coded modulation over a Rayleigh channel could be improved by using bit wise interleaving after the encoder rather than using symbol wise interleaving after the modulator. The order of diversity for any coded system with a symbol interleaver is the minimum number of distinct symbols between codewords. Preventing parallel transitions and increasing the constraint length of the code are the only ways of increasing diversity. However bit-wise interleaving results in the diversity dictated by the smallest number of distinct bits, rather than channel symbols, along any error event. BICM because of its improved code diversity over fast fading channels has made its place in almost all the upcoming wireless communication standards as IEEE 802.11n [3], IEEE 802.16m [4] and 3rd generation partnership project long term evolution (3GPP LTE) [5].

This improved diversity comes at the cost of increased detection complexity. For single input single output (SISO) coded systems using symbol interleaver, the symbol metric for maximum likelihood (ML) detection is based on the calculation of minimum distance between the received symbol and $M$ constellation points on the complex plane ( $M$-ary QAM alphabet). However in the case of bit interleaving, same number of computations $\left(2^{\log M}\right)$ are involved for each bit metric which is also called as log-likelihood ratio (LLR). This complexity renders real time implementation of BICM based systems in existing hardware quite intricate especially for large sized constellations. Moreover the addition of spatial dimension (MIMO) as being advocated for almost all upcoming wireless systems is going to increase the computational complexity to $2^{\log (M) \times n_{t}}$ for each LLR where $n_{t}$ is the number of transmit antennas/ spatial streams. Requisite antenna spacing and complexity constraints have restricted $n_{t}$ to 4 for next generation wireless systems whereas it is reduced to dual streams in most scenarios [4] [5].

Research community has been trying to find simplified receiver structures for such SISO and MIMO systems in accordance with the existing hardware limitations. A set of approximated bit metrics using the LLR for each bit are given in [6] for SISO bit interleaved OFDM systems for QAM16 and QAM64 constellations. Later in [7] authors showed that for Gray encoded BICM systems, the minimum distance calculation for each bit LLR can be reduced from $M$ points on complex constellation to $\sqrt{M}$ points on real line. Our contribution in this paper is to show that the soft decision bit metrics for the ML decoder for BICM SISO systems can be significantly simplified to a scaled version of the matched filter (MF) output. This simplification is aided by the decoupling of real and imaginary parts of the metric. Resultant bit metrics need no computation of minimum distances and their comparisons. The complexity is maintained at almost the same level for all constellations as it involves only the computation of MF output. This fundamentally reduces the number of calculations needed for each bit LLR without compromising the performance. We also tabulate the implementation advantages of the simplified bit metrics compared to the original ones.

For BICM MIMO systems, though many sophisticated 


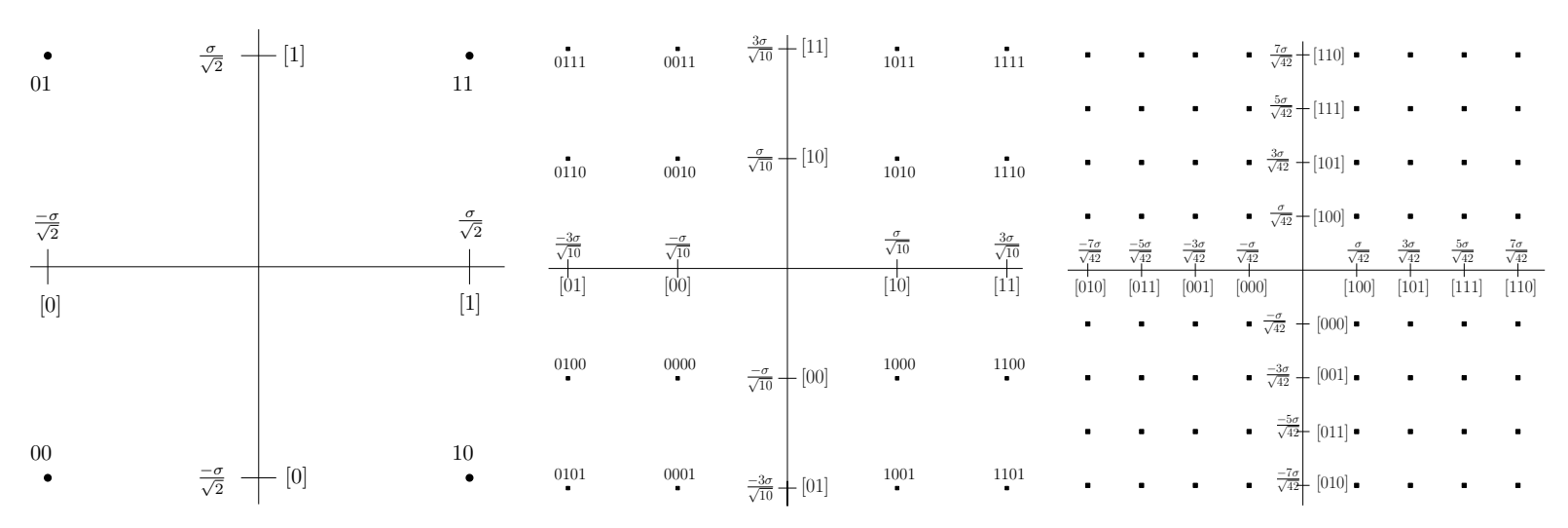

Fig. 1. Normalized constellations of QPSK, QAM16 and QAM64 with Gray mapping.

methods as lattice reduction and sphere decoding [8] have been proposed in the literature which find the ML solution with high probability, but these methods are in general still computationally complex for real-time implementation. Moreover not much attention has been paid to the practical low dimensional MIMO systems. We further propose in this paper a simplified MF based bit metric for dual stream BICM case which successfully decouples two streams and subsequently reduces one complex dimension of the system. The proposed bit metric therefore reduces the complexity of dual stream case to the complexity of single stream detection. These low complexity metrics for SISO and MIMO systems being based on MF outputs and devoid of any division operation are suitable for hardware implementation. These metrics are being incorporated in the receiver structure of Eurecom OpenAirInterface platform, which is an experimental real-time opensource hardware and software platform for future wireless networks [9] (www.openairinterface.org).

Regarding notations, we will use lowercase or uppercase letters for scalars, lowercase boldface letters for vectors and uppercase boldface letters for matrices. $(.)^{*}$ and $(.)^{\dagger}$ indicate conjugate and conjugate transpose operations respectively. $|$. indicates norm of a complex scalar or absolute value of a real scalar while $\|$.$\| indicates norm of a vector. (.)_{R}$ denotes real part of a complex number while $(.)_{I}$ indicates its imaginary part. Note that all $\log$ are to the base 2. This paper is mainly divided into two parts. In first part we propose the simplified metrics for BICM SISO systems while second part is dedicated to the simplified metrics for dual stream BICM case. Performance of the proposed metrics is verified by the simulation results and conclusions are presented at the end of the paper.

\section{BICM SISO SYSTEM}

\section{A. System Model}

We consider the downlink of a cellular system with $n_{t}$ antennas at the base station (BS) and one antenna at the mobile station (MS). Keeping in view the upcoming wireless standards as $802.16 \mathrm{~m} \mathrm{[4]} \mathrm{and} \mathrm{3GPP} \mathrm{LTE} \mathrm{[5],} \mathrm{we} \mathrm{assume}$ that the BS uses BICM based OFDM system for downlink transmission. We further assume antenna cycling at the BS [10] with each stream being transmitted by one antenna in any dimension. The antenna is randomly assigned per dimension so that the stream sees all degrees of freedom of the channel. $x$ is the symbol over a signal set $\chi \subseteq \mathcal{C}$ with a Gray labeling map $\mu:\{0,1\}^{\log _{2}|\chi|} \rightarrow \chi$. During the transmission at BS, code sequence $\mathbf{c}$ is interleaved by $\pi$ and then is mapped onto the signal sequence $\mathbf{x} \in \chi$. Bit interleaver can be modeled as $\pi: k^{\prime} \rightarrow(k, i)$ where $k^{\prime}$ denotes the original ordering of the coded bits $c_{k^{\prime}}, k$ denotes the time ordering of the signal $x_{k}$ and $i$ indicates the position of the bit $c_{k^{\prime}}$ in the symbol $x_{k}$. We assume that cyclic prefix (CP) of appropriate length is added to the OFDM symbols at the BS. Cascading IFFT at the BS and FFT at the MS with CP extension, transmission at the $k$-th frequency tone can be expressed as

$$
y_{k}=h_{k} x_{k}+z_{k}, \quad k=1,2, \cdots, K
$$

where $h_{k}$ is the channel at the $k$-th frequency tone. Each subcarrier corresponds to a symbol from the constellation map $\chi . y_{k}, z_{k} \in \mathcal{C}$ are the received symbols and circularly symmetric complex white Gaussian noise of double-sided power spectral density $N_{0} / 2 . h_{k} \in \mathcal{C}$ characterizes flat fading channel response from transmitting antenna to MS at $k$-th subcarrier. The complex symbol $x_{k}$ has the variance $\sigma^{2}$.

Let $\chi_{c_{k^{\prime}}}^{i}$ denotes the subset of all signals $x \in \chi$ whose label has the value $c_{k^{\prime}} \in\{0,1\}$ in position $i$. Then the ML bit metric can be given by

$$
\lambda^{i}\left(y_{k}, c_{k^{\prime}}\right)=\max _{x \in \chi_{c_{k^{\prime}}^{i}}^{i}} \log p\left(y_{k} \mid x, h_{k}\right)
$$

The original ML metric involves summation instead of the maximum operation and the above metric is commonly referred as max log MAP metric. However the complexity lies in the computation of the terms in the metric rather than their summation or maximization operation. The ML decoder at the receiver can make decisions according to the rule

$$
\hat{\mathbf{c}}=\arg \max _{\mathbf{c} \in \mathbb{C}} \sum_{k^{\prime}} \lambda^{i}\left(y_{k}, c_{k^{\prime}}\right)
$$

where $\mathbb{C}$ defines the code book. This metric incorporates different ordering of the bits before and after the interleaver 

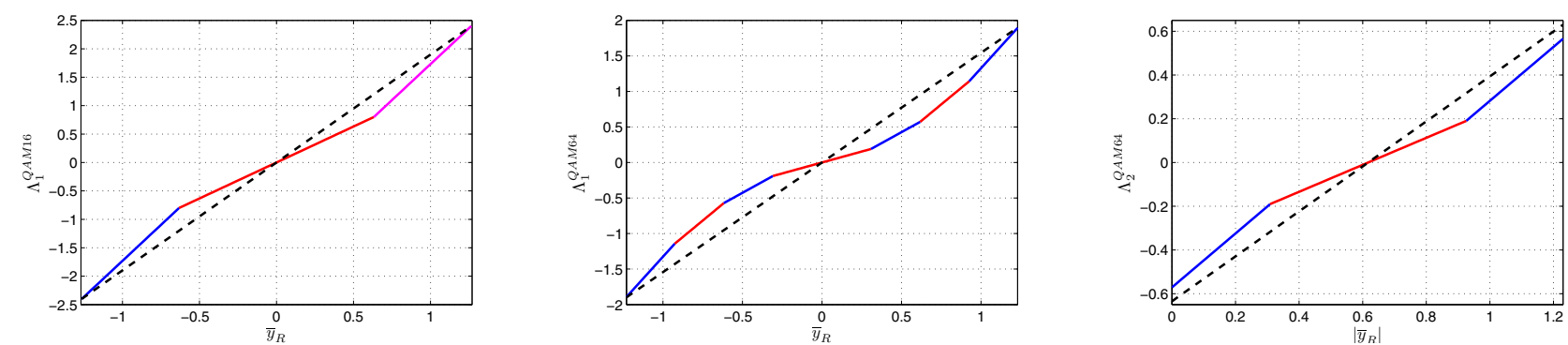

Fig. 2. Continuous line indicates the exact bit metric while the dashed line indicates the approximated bit metric. Note that $|h|$ and $\sigma$ are taken as 1 .

at the transmitter in decoding, by associating a contribution to the metric for each bit associated with the symbol received while that bit is transmitted. The bit LLR can be given as

$$
\begin{aligned}
& \Lambda_{i}\left(y_{k}, c_{k^{\prime}}\right)=\lambda^{i}\left(y_{k}, c_{k^{\prime}}=1\right)-\lambda^{i}\left(y_{k}, c_{k^{\prime}}=0\right) \\
& =\max _{x \in \chi_{k_{k^{\prime}}^{i}=1}^{i}}\left\{\frac{-1}{N_{0}}\left|y_{k}-h_{k} x\right|^{2}\right\}-\max _{x \in \chi_{c_{k^{\prime}}^{i}=0}^{i}}\left\{\frac{-1}{N_{0}}\left|y_{k}-h_{k} x\right|^{2}\right\}
\end{aligned}
$$

\section{B. Simplified Bit Metrics}

In this section, we propose simplifications and approximations for (4) and state bit metrics for QPSK, QAM16 and QAM64 constellations. Using same techniques, simplified bit metrics can also be derived for larger sized constellations. Due to space constraints, we drop the frequency and interleaving indices. Rewriting (4) in terms of real and imaginary parts

$$
\begin{aligned}
\Lambda_{i}= & \max _{x \in \chi_{1}^{i}}\left\{2 \bar{y}_{R} x_{R}+2 \bar{y}_{I} x_{I}-|h|^{2} x_{R}^{2}-|h|^{2} x_{I}^{2}\right\} \\
& -\max _{x \in \chi_{0}^{i}}\left\{2 \bar{y}_{R} x_{R}+2 \bar{y}_{I} x_{I}-|h|^{2} x_{R}^{2}-|h|^{2} x_{I}^{2}\right\} \\
= & \max _{x_{R} \in \chi_{1}^{i}}\left\{2 \bar{y}_{R} x_{R}-|h|^{2} x_{R}^{2}\right\}+\max _{x_{I} \in \chi_{1}^{i}}\left\{2 \bar{y}_{I} x_{I}-|h|^{2} x_{I}^{2}\right\} \\
& -\max _{x_{R} \in \chi_{0}^{i}}\left\{2 \bar{y}_{R} x_{R}-|h|^{2} x_{R}^{2}\right\}-\max _{x_{I} \in \chi_{0}^{i}}\left\{2 \bar{y}_{I} x_{I}-|h|^{2} x_{I}^{2}\right\}
\end{aligned}
$$

where $\bar{y}_{k}=h_{k}^{*} y_{k}$ is the MF output. $x_{R}$ is the real and $x_{I}$ is the imaginary part of $x$ while $\bar{y}_{R}$ and $\bar{y}_{I}$ are real and imaginary parts of $\bar{y}$. Note that $x_{R} \in \chi_{1}^{i}$ represent the real parts of those constellation points of $\chi$ where the bit at position $i$ is 1. The metric (5) decouples the real and imaginary parts. Let $m=\log M$ denotes the number of bits per symbol. Then as per the Gray encoding shown in Fig. 1 for $i=1,2, \cdots, m / 2$, $x_{I} \in \chi_{1}^{i}=x_{I} \in \chi_{0}^{i}$ so the LLR is written as

$$
\Lambda_{i}=\max _{x_{R} \in \chi_{1}^{i}}\left\{2 \bar{y}_{R} x_{R}-|h|^{2} x_{R}^{2}\right\}-\max _{x_{R} \in \chi_{0}^{i}}\left\{2 \bar{y}_{R} x_{R}-|h|^{2} x_{R}^{2}\right\}
$$

whereas for $i=m / 2+1, \cdots, m, x_{R} \in \chi_{1}^{i}=x_{R} \in \chi_{0}^{i}$, so the LLR can be written as

$$
\Lambda_{i}=\max _{x_{I} \in \chi_{1}^{i}}\left\{2 \bar{y}_{I} x_{I}-|h|^{2} x_{I}^{2}\right\}-\max _{x_{I} \in \chi_{0}^{i}}\left\{2 \bar{y}_{I} x_{I}-|h|^{2} x_{R}^{2}\right\}
$$

Basing on this, LLR for the first bit for QPSK is given as

$$
\begin{aligned}
\Lambda_{1}^{\mathrm{QPSK}} & =\max _{\substack{x_{R}=\frac{\sigma}{\sqrt{2}} \\
x_{I}}}\left\{\bar{y}_{R} x_{R}+\bar{y}_{I} x_{I}\right\}-\max _{\substack{x_{R}=\frac{-\sigma}{\sqrt{2}} \\
x_{I}}}\left\{\bar{y}_{R} x_{R}+\bar{y}_{I} x_{I}\right\} \\
& =\max _{x_{I}}\left\{\bar{y}_{I} x_{I}\right\}-\max _{x_{I}}\left\{\bar{y}_{I} x_{I}\right\}+\sqrt{2} \sigma \bar{y}_{R} \\
& =\sqrt{2} \sigma \bar{y}_{R}
\end{aligned}
$$

whereas for the second bit, LLR is given as

$$
\begin{aligned}
\Lambda_{2}^{\mathrm{QPSK}} & =\max _{\substack{x_{I}=\frac{\sigma}{\sqrt{2}} \\
x_{R}}}\left\{\bar{y}_{R} x_{R}+\bar{y}_{I} x_{I}\right\}-\max _{\substack{x_{I}=\frac{-\sigma}{\sqrt{2}} \\
x_{R}}}\left\{\bar{y}_{R} x_{R}+\bar{y}_{I} x_{I}\right\} \\
& =\sqrt{2} \sigma \bar{y}_{I}
\end{aligned}
$$

Similarly for QAM16, the first bit LLR is written as

$$
\begin{aligned}
\Lambda_{1}^{\mathrm{QAM} 1} \stackrel{\underline{6}}{=} \max _{x_{R}=\frac{\sigma}{\sqrt{10}}, \frac{3 \sigma}{\sqrt{10}}}\left\{2 \bar{y}_{R} x_{R}-|h|^{2} x_{R}^{2}\right\}-\max _{x_{R}=\frac{-\sigma}{\sqrt{10}}, \frac{-3 \sigma}{\sqrt{10}}}\left\{2 \bar{y}_{R} x_{R}-|h|^{2} x_{R}^{2}\right\} \\
= \begin{cases}\frac{8 \sigma}{\sqrt{10}} \bar{y}_{R}+\frac{8|h|^{2} \sigma^{2}}{10} & \text { if } \bar{y}_{R}<-\frac{2 \sigma|h|^{2}}{\sqrt{10}} \\
\frac{4 \sigma}{\sqrt{10}} \bar{y}_{R} & \text { if }-\frac{2 \sigma|h|^{2}}{\sqrt{10}}<\bar{y}_{R}<\frac{2 \sigma|h|^{2}}{\sqrt{10}} \\
\frac{8 \sigma}{\sqrt{10}} \bar{y}_{R}-\frac{8|h|^{2} \sigma^{2}}{10} & \text { if } \bar{y}_{R}>\frac{2 \sigma|h|^{2}}{\sqrt{10}}\end{cases} \\
\approx \frac{20 \sigma}{3 \sqrt{10}} \bar{y}_{R}
\end{aligned}
$$

where we have introduced a little suboptimality in the last step by averaging over three possible bit metrics. The approximated bit metric is indicated by the dotted line in Fig. 2 (left figure) while the continuous line shows the exact bit metric (three regions). The LLR for the second bit is written as

$$
\begin{aligned}
& \Lambda_{2}^{\mathrm{QAM}} \underset{\underline{\underline{x_{R}}}}{\underline{\underline{6}}=\frac{3 \sigma}{\sqrt{10}}, \frac{-3 \sigma}{\sqrt{10}}}\left\{2 \bar{y}_{R} x_{R}-|h|^{2} x_{R}^{2}\right\}-\max _{x_{R}=\frac{\sigma}{\sqrt{10}}, \frac{-\sigma}{\sqrt{10}}}\left\{2 \bar{y}_{R} x_{R}-|h|^{2} x_{R}^{2}\right\} \\
& \quad=\frac{4 \sigma}{\sqrt{10}}\left|\bar{y}_{R}\right|-\frac{8|h|^{2} \sigma^{2}}{10}
\end{aligned}
$$

LLRs for the third and fourth bit are similar to the first and second bit respectively except that $\bar{y}_{R}$ is replaced by $\bar{y}_{I}$. Two LLRs are exact while two LLRs have slight suboptimality for 
QAM16. For QAM64, LLR for the first bit is given as

$$
\begin{aligned}
& \Lambda_{1}^{\text {QAM64 }}=\max _{x_{R}=\frac{\sigma}{\sqrt{42}}, \frac{3 \sigma}{\sqrt{42}}, \frac{5 \sigma}{\sqrt{42}}, \frac{7 \sigma}{\sqrt{42}}}\left\{2 \bar{y}_{R} x_{R}-|h|^{2} x_{R}^{2}\right\}
\end{aligned}
$$

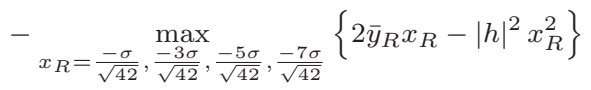

$$
\begin{aligned}
& = \begin{cases}\frac{16 \sigma}{\sqrt{42}} \bar{y}_{R}+\frac{48|h|^{2} \sigma^{2}}{42} & \text { if } \bar{y}_{R}<-\frac{6 \sigma|h|^{2}}{\sqrt{42}} \\
\frac{12 \sigma}{\sqrt{42}} \bar{y}_{R}+\frac{24|h|^{2} \sigma^{2}}{42} & \text { if }-\frac{6 \sigma|h|^{2}}{\sqrt{42}}<\bar{y}_{R}<-\frac{4 \sigma|h|^{2}}{\sqrt{42}} \\
\frac{8 \sigma}{\sqrt{42}} \bar{y}_{R}+\frac{8|h|^{2} \sigma^{2}}{42} & \text { if }-\frac{4 \sigma|h|^{2}}{\sqrt{42}}<\bar{y}_{R}<-\frac{2 \sigma|h|^{2}}{\sqrt{42}} \\
\frac{4 \sigma}{\sqrt{42}} \bar{y}_{R} & \text { if }-\frac{2 \sigma|h|^{2}}{\sqrt{42}}<\bar{y}_{R}<\frac{2 \sigma|h|^{2}}{\sqrt{42}} \\
\frac{8 \sigma}{\sqrt{42}} \bar{y}_{R}-\frac{8|h|^{2} \sigma^{2}}{42} & \text { if } \frac{2 \sigma|h|^{2}}{\sqrt{42}}<\bar{y}_{R}<\frac{4 \sigma|h|^{2}}{\sqrt{42}} \\
\frac{12 \sigma}{\sqrt{42}} \bar{y}_{R}-\frac{24|h|^{2} \sigma^{2}}{42} & \text { if } \frac{4 \sigma|h|^{2}}{\sqrt{42}}<\bar{y}_{R}<\frac{6 \sigma||^{2}}{\sqrt{42}} \\
\frac{16 \sigma}{\sqrt{42}} \bar{y}_{R}-\frac{48|h|^{2} \sigma^{2}}{42} & \text { if } \frac{6 \sigma|h|^{2}}{\sqrt{42}}<\bar{y}_{R}\end{cases} \\
& \approx \frac{10 \sigma}{\sqrt{42}} \bar{y}_{R}
\end{aligned}
$$

Again we have introduced a slight suboptimality in the last step by averaging over seven possible bit metrics as shown in Fig. 2 (center figure). Similarly for the second bit, LLR is written as

$$
\begin{aligned}
& \Lambda_{2}^{\text {QAM64 }}=\max _{x_{R}=\frac{5 \sigma}{\sqrt{42}}, \frac{7 \sigma}{\sqrt{42}}, \frac{-5 \sigma}{\sqrt{42}}, \frac{-7 \sigma}{\sqrt{42}}}\left\{2 \bar{y}_{R} x_{R}-|h|^{2} x_{R}^{2}\right\}
\end{aligned}
$$

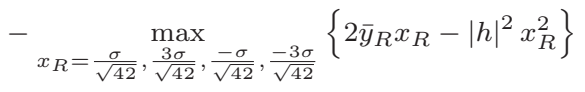

$$
\begin{aligned}
& = \begin{cases}\frac{8 \sigma}{\sqrt{42}}\left|\bar{y}_{R}\right|-\frac{24|h|^{2} \sigma^{2}}{42} & \text { if }\left|\bar{y}_{R}\right|<\frac{2 \sigma|h|^{2}}{\sqrt{42}} \\
\frac{4 \sigma}{\sqrt{42}}\left|\bar{y}_{R}\right|-\frac{16|h|^{2} \sigma^{2}}{42} & \text { if } \frac{2 \sigma|h|^{2}}{\sqrt{42}}<\left|\bar{y}_{R}\right|<\frac{6 \sigma|h|^{2}}{\sqrt{42}} \\
\frac{8 \sigma}{\sqrt{42}}\left|\bar{y}_{R}\right|-\frac{40|h|^{2} \sigma^{2}}{42} & \text { if } \frac{6 \sigma|h|^{2}}{\sqrt{42}}<\left|\bar{y}_{R}\right|\end{cases} \\
& \approx \frac{20 \sigma}{3 \sqrt{42}}\left|\bar{y}_{R}\right|-\frac{80|h|^{2} \sigma^{2}}{3 \times 42}
\end{aligned}
$$

The suboptimality introduced in the last step is shown in Fig. 2 (right figure). For third bit, LLR is given as

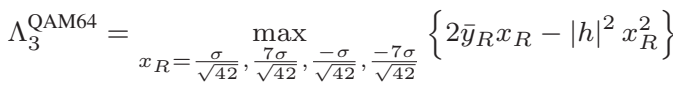

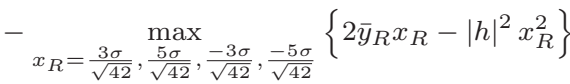

$$
\begin{aligned}
& = \begin{cases}\frac{-4 \sigma}{\sqrt{42}}\left|\bar{y}_{R}\right|+\frac{8|h|^{2} \sigma^{2}}{42} & \text { if }\left|\bar{y}_{R}\right|<\frac{4 \sigma|h|^{2}}{\sqrt{42}} \\
\frac{4 \sigma}{\sqrt{42}}\left|\bar{y}_{R}\right|-\frac{24|h|^{2} \sigma^{2}}{42} & \text { if }\left|\bar{y}_{R}\right|>\frac{4 \sigma|h|^{2}}{\sqrt{42}}\end{cases} \\
& =\left|\frac{4 \sigma}{\sqrt{42}}\right| \bar{y}_{R}\left|-\frac{16|h|^{2} \sigma^{2}}{42}\right|-\frac{8|h|^{2} \sigma^{2}}{42}
\end{aligned}
$$

LLRs for fourth, fifth and sixth bits are similar to first, second and third bits respectively with $\bar{y}_{R}$ being replaced by $\bar{y}_{I}$. Amongst six bits of QAM64, four bits have approximated LLRs whereas two bits have exact LLRs. Using similar techniques, bit LLRs for higher sized constellations can be easily found. The complexity analysis of these bit metrics

TABLE I

COMPUTATIONAL COMPLEXITY FOR CALCULATION OF LLRS IN ONE SYMBOL IN SISO SYSTEM

\begin{tabular}{|c|c|c|c|c|c|c|}
\hline$M$-ary & \multicolumn{2}{|c|}{ Multiplications } & \multicolumn{2}{c|}{ Additions } & \multicolumn{2}{c|}{ Comparisons } \\
\hline & original & simplified & original & simplified & original & simplified \\
4 & 16 & 1 & 8 & 0 & 8 & 0 \\
16 & 128 & 2 & 64 & 0 & 64 & 0 \\
64 & 768 & 2 & 384 & 0 & 384 & 0 \\
\hline
\end{tabular}

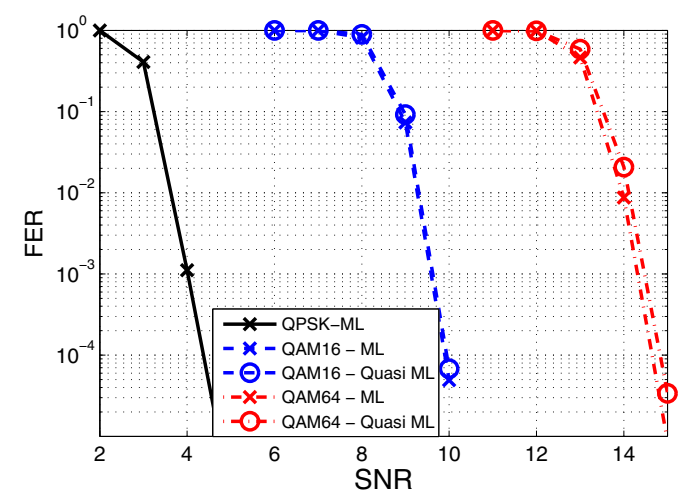

Fig. 3. SISO System using punctured rate $1 / 2$ turbo code of 3GPP LTE. Decoding iterations are 5 .

in terms of complex operations (complex multiplications, complex additions) is tabulated in table.I. Number of complex multiplications for the simplified metrics come from the computation of MF output and the channel norm. Note that where the complexity of original metrics increases exponentially in $m$, the complexity of simplified metrics is maintained at almost the same level for all constellations.

\section{Simulation Results}

We consider a BICM SISO OFDM system using the rate$1 / 2$ punctured turbo code $^{1}$ proposed for 3 GPP LTE [5]. The channel has iid Gaussian matrix entries with unit variance and the effect of antenna cycling is simulated by the independent generation of channel each time instant. Perfect CSI at the receiver is assumed. We consider the original max log MAP bit metrics (ML) and the simplified metrics. Due to the approximations involved, we call the simplified bit metric approach as Quasi-ML approach. We focus on frame error rates where the frame length is fixed to 1296 information bits as per 802.11n [3]. Note that for QPSK, simplified bit metrics are same as ML metrics. Fig. 3 shows that there is no degradation in the performance with the simplified bit metrics for QAM16 while there is a slight degradation in the performance for QAM64 case. It is attributed to four approximated bit metrics in case of QAM64 compared to two approximated bit metrics for QAM16.

\section{BICM MIMO SYSTEM}

\section{A. System Model}

Consider a $n_{t} \times n_{r}$ BICM MIMO OFDM system $\left(n_{t} \geq 2\right)$ with 2 spatial streams as shown in Fig. 4. We effectively reduce this to $2 \times n_{r}$ system by antenna cycling at the transmitter with each stream being transmitted by one antenna in any dimension. Two spatial streams are $\mathbf{x}_{1}$ and $\mathbf{x}_{2}$ and they can be of uniform or nonuniform rates. $x_{1}$ is the symbol of $\mathbf{x}_{1}$ over a signal set $\chi_{1} \subseteq \mathcal{C}$ with a Gray labeling map and $x_{2}$ is

\footnotetext{
${ }^{1}$ The LTE turbo decoder design was performed using the coded modulation library www.iterativesolutions.com
} 


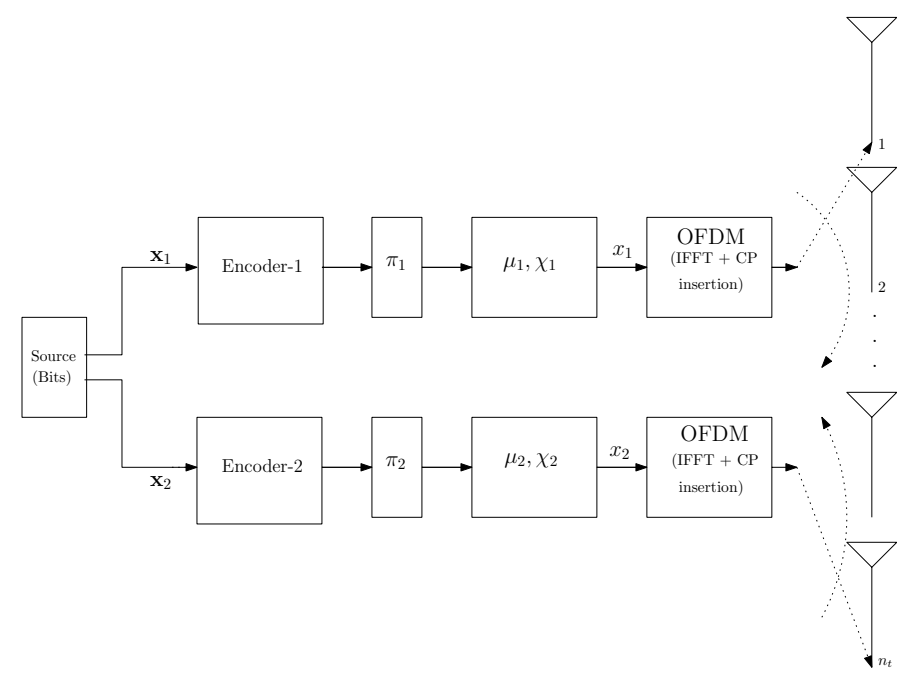

Fig. 4. Block diagram of the transmitter of $n_{t} \times n_{r}$ BICM MIMO OFDM system. $\pi_{1}$ denotes random interleaver, $\mu_{1}$ labeling map and $\chi_{1}$ signal set for $\mathbf{x}_{1}$.

the symbol of $\mathbf{x}_{2}$ over signal set $\chi_{2}$. Transmission at the $k$-th frequency tone can be expressed as

$$
\mathbf{y}_{k}=\mathbf{h}_{1, k} x_{1, k}+\mathbf{h}_{2, k} x_{2, k}+\mathbf{z}_{k}, \quad k=1,2, \cdots, K
$$

where $\mathbf{y}_{k}, \mathbf{z}_{k} \in \mathbb{C}^{n_{r}}$ are the vectors of received symbols and circularly symmetric complex white Gaussian noise of doublesided power spectral density $N_{0} / 2$ at $n_{r}$ receive antennas. $\mathbf{h}_{1, k} \in \mathbb{C}^{n_{r}}$ is the vector characterizing flat fading channel response from first transmitting antenna to $n_{r}$ receive antennas at $k$-th subcarrier. The complex symbols $x_{1, k}$ and $x_{2, k}$ of the two streams are assumed to be of variances $\sigma_{1}^{2}$ and $\sigma_{2}^{2}$ respectively. The ML bit metric for the bit $c_{k^{\prime}}$ for first stream is given as

$$
\lambda_{1}^{i}\left(\mathbf{y}_{k}, c_{k^{\prime}}\right) \approx \max _{x_{1} \in \chi_{1, c_{k^{\prime}}}^{i}, x_{2} \in \chi_{2}} \frac{-1}{N_{0}}\left\|\mathbf{y}_{k}-\mathbf{h}_{1, k} x_{1}-\mathbf{h}_{2, k} x_{2}\right\|^{2}
$$

The complexity of this bit metric is $\mathcal{O}\left(\left|\chi_{1}\right|\left|\chi_{2}\right|\right)$

\section{B. Simplified Bit Metrics}

For the ease of notation, we drop the frequency and interleaving indices so (15) can be rewritten as

$$
\begin{aligned}
\lambda_{1}^{i}(\mathbf{y}, c) \approx \max _{x_{1} \in \chi_{1, c}^{i}, x_{2} \in \chi_{2}} & \frac{-1}{N_{0}}\left\|\mathbf{y}-\mathbf{h}_{1} x_{1}-\mathbf{h}_{2} x_{2}\right\|^{2} \\
=\max _{x_{1} \in \chi_{1, c}^{i}, x_{2} \in \chi_{2}}\{ & -\left\|\mathbf{h}_{1}\right\|^{2} x_{1, R}^{2}-\left\|\mathbf{h}_{1}\right\|^{2} x_{1, I}^{2}+2 y_{1, R} x_{1, R}+2 y_{1, I} x_{1, I} \\
& -2\left(p_{12, R} x_{1, R}+p_{12, I} x_{1, I}-y_{2, R}\right) x_{2, R}-\left\|\mathbf{h}_{2}\right\|^{2} x_{2, R}^{2} \\
& \left.-2\left(p_{12, R} x_{1, I}-p_{12, I} x_{1, R}-y_{2, I}\right) x_{2, I}-\left\|\mathbf{h}_{2}\right\|^{2} x_{2, I}^{2}\right\}
\end{aligned}
$$

where $y_{1}=\mathbf{h}_{1}^{\dagger} \mathbf{y}$ and $y_{2}=\mathbf{h}_{2}^{\dagger} \mathbf{y}$ are the MF outputs for first and second stream respectively while $p_{12}=\mathbf{h}_{1}^{\dagger} \mathbf{h}_{2}$ is the cross correlation between first and second channel. Evaluation of (17) implies that for each constellation point of $x_{1} \in \chi_{1, c}^{i}$, we need to find the values of $x_{2, R} \in \chi_{2}$ and $x_{2, I} \in \chi_{2}$ which maximize the metric (17). To reduce the complexity, we need to decouple $x_{1}$ and $x_{2}$.

First we consider equal energy alphabets e.g. QPSK-QPSK i.e. both $x_{1}$ and $x_{2}$ belong to QPSK. Being of same magnitude, we need to look only at the signs of $x_{2, R}$ and $x_{2, I}$ for maximizing (17). Evidently maximization implies that they must be in the opposite directions of $\left(p_{12, R} x_{1, R}+p_{12, I} x_{1, I}-y_{2, R}\right)$ and $\left(p_{12, R} x_{1, I}-p_{12, I} x_{1, R}-y_{2, I}\right)$ respectively. So the bit metric is written as

$$
\begin{aligned}
& \lambda_{1}^{i}(\mathbf{y}, c)=\max _{x_{1} \in \chi_{1, c}^{i}}\left\{\left|p_{12, R} x_{1, R}+p_{12, I} x_{1, I}-y_{2, R}\right|\left|x_{2, R}\right|\right. \\
& \left.\quad+\left|p_{12, R} x_{1, I}-p_{12, I} x_{1, R}-y_{2, I}\right|\left|x_{2, I}\right|+y_{1, R} x_{1, R}+y_{1, I} x_{1, I}\right\}
\end{aligned}
$$

which reduces one complex dimension of the system.

For the case of non equal energy alphabets, we need to look both at the magnitudes and the signs of $x_{2, R}$ and $x_{2, I}$. Regarding signs, the earlier argument also holds for non equal energy alphabets. The magnitudes of $x_{2, R}$ and $x_{2, I}$ which maximize (17) are given as

$$
\begin{aligned}
\left|x_{2, R}\right| & =\frac{\left|p_{12, R} x_{1, R}+p_{12, I} x_{1, I}-y_{2, R}\right|}{\left\|\mathbf{h}_{2}\right\|^{2}} \\
\left|x_{2, I}\right| & =\frac{\left|p_{12, R} x_{1, I}-p_{12, I} x_{1, R}-y_{2, I}\right|}{\left\|\mathbf{h}_{2}\right\|^{2}}
\end{aligned}
$$

We can easily find the discrete points of constellations from these continuous values. For the case of QAM16-QAM16, these magnitudes can be found in one operation i.e.

$$
\begin{array}{r}
\left|x_{2, R}\right|=\sigma_{2} \frac{1}{\sqrt{10}}\left(2+(-1)^{I\left(\left|p_{12, R} x_{1, R}+p_{12, I} x_{1, I}-y_{2, R}\right|<\sigma_{2} \frac{2\left\|\mathbf{h}_{\mathbf{2}}\right\|^{2}}{\sqrt{10}}\right)}\right) \\
\left|x_{2, I}\right|=\sigma_{2} \frac{1}{\sqrt{10}}\left(2+(-1)^{I\left(\left|p_{12, R} x_{1, I}-p_{12, I} x_{1, R}-y_{2, I}\right|<\sigma_{2} \frac{2\left\|\mathbf{h}_{\mathbf{2}}\right\|^{2}}{\sqrt{10}}\right)}\right)
\end{array}
$$

where $I($.$) is the indicator function defined as$

$$
I(a<b)= \begin{cases}1 & \text { if } a<b \\ 0 & \text { otherwise }\end{cases}
$$

Knowing these magnitudes, the bit metric is written as

$$
\begin{aligned}
\lambda_{1}^{i}(\mathbf{y}, c) \approx & \max _{x_{1} \in \chi_{1, c}^{i}}\left\{-\left\|\mathbf{h}_{1}\right\|^{2} x_{1, R}^{2}-\left\|\mathbf{h}_{1}\right\|^{2} x_{1, I}^{2}+2 y_{1, R} x_{1, R}+2 y_{1, I} x_{1, I}\right. \\
& +2\left|p_{12, R} x_{1, R}+p_{12, I} x_{1, I}-y_{2, R}\right| \times\left|x_{2, R}\right|-\left\|\mathbf{h}_{2}\right\|^{2}\left|x_{2, R}\right|^{2} \\
& \left.+2\left|p_{12, R} x_{1, I}-p_{12, I} x_{1, R}-y_{2, I}\right| \times\left|x_{2, I}\right|-\left\|\mathbf{h}_{2}\right\|^{2}\left|x_{2, I}\right|^{2}\right\}
\end{aligned}
$$

So the complexity of the metric for first stream reduces to $\mathcal{O}\left(\left|\chi_{1}\right|\right)$.

For QAM64-QAM64, bit metric is same as (21) while $\left|x_{2, R}\right|$ and $\left|x_{2, I}\right|$ are given by (16) on the top of the next page where $\psi_{R}=p_{12, R} x_{1, R}+p_{12, I} x_{1, I}-y_{2, R}$ and $\psi_{I}=p_{12, R} x_{1, I}-$ $p_{12, I} x_{1, R}-y_{2, I}$. Note that $\|$ indicates OR operation.

We take another example once the two streams have nonuniform rate i.e. QAM16-QPSK. So then the bit metric for 


$$
\begin{aligned}
& \left.\left|x_{2, R}\right|=\sigma_{2} \frac{1}{\sqrt{42}}\left\{4+(-1)^{I\left(\left|\Psi_{R}\right|<\sigma_{2}\right.} \frac{4\left\|\mathbf{h}_{2}\right\|^{2}}{\sqrt{42}}\right)+2(-1)^{I\left(\left|\Psi_{R}\right|<\sigma_{2} \frac{2\left\|\mathbf{h}_{2}\right\|^{2}}{\sqrt{42}}\right)}\left[I\left(\left|\Psi_{R}\right|<\sigma_{2} \frac{2\left\|\mathbf{h}_{2}\right\|^{2}}{\sqrt{42}}\right) \| I\left(\left|\Psi_{R}\right|>\sigma_{2} \frac{6\left\|\mathbf{h}_{2}\right\|^{2}}{\sqrt{42}}\right)\right]\right\} \\
& \left|x_{2, I}\right|=\sigma_{2} \frac{1}{\sqrt{42}}\left\{4+(-1)^{I\left(\left|\Psi_{I}\right|<\sigma_{2} \frac{4\left\|\mathbf{h}_{2}\right\|^{2}}{\sqrt{42}}\right)}+2(-1)^{I\left(\left|\Psi_{I}\right|<\sigma_{2} \frac{2\left\|\mathbf{h}_{2}\right\|^{2}}{\sqrt{42}}\right)}\left[I\left(\left|\Psi_{I}\right|<\sigma_{2} \frac{2\left\|\mathbf{h}_{2}\right\|^{2}}{\sqrt{42}}\right) \| I\left(\left|\Psi_{I}\right|>\sigma_{2} \frac{6\left\|\mathbf{h}_{2}\right\|^{2}}{\sqrt{42}}\right)\right]\right\}
\end{aligned}
$$

first stream (QAM16) is given as

$$
\begin{aligned}
\lambda_{1}^{i}(\mathbf{y}, c)=\max _{x_{1} \in \chi_{1, c}^{i}}\left\{-\left\|\mathbf{h}_{1}\right\|^{2} x_{1, R}^{2}-\left\|\mathbf{h}_{1}\right\|^{2} x_{1, I}^{2}+2 y_{1, R} x_{1, R}+2 y_{1, I} x_{1, I}\right. \\
+2\left|p_{12, R} x_{1, R}+p_{12, I} x_{1, I}-y_{2, R}\right|\left|x_{2, R}\right| \\
\left.+2\left|p_{12, R} x_{1, I}-p_{12, I} x_{1, R}-y_{2, I}\right|\left|x_{2, I}\right|\right\}
\end{aligned}
$$

whereas for second stream i.e. QPSK (Note that $x_{1}=$ QAM16 and $\left.x_{2}=\mathrm{QPSK}\right)$, the bit metric is given by

$$
\begin{aligned}
\lambda_{2}^{i}(\mathbf{y}, c)=\max _{x_{2} \in \chi_{2, c}^{i}\{}\{ & \left\|\mathbf{h}_{1}\right\|^{2} x_{1, R}^{2}-\left\|\mathbf{h}_{1}\right\|^{2} x_{1, I}^{2}+2 y_{2, R} x_{2, R}+2 y_{2, I} x_{2, I} \\
& +2\left|p_{12, R} x_{2, R}-p_{12, I} x_{2, I}-y_{1, R}\right|\left|x_{1, R}\right| \\
& \left.+2\left|p_{12, R} x_{2, I}+p_{12, I} x_{2, R}-y_{1, I}\right|\left|x_{1, I}\right|\right\}
\end{aligned}
$$

where $\left|x_{1, R}\right|$ and $\left|x_{1, I}\right|$ are given by

$$
\begin{array}{r}
\left|x_{1, R}\right|=\sigma_{1} \frac{1}{\sqrt{10}}\left(2+(-1)^{\left.I\left(\left|p_{12, R} x_{2, R}-p_{12, I} x_{2, I}-y_{1, R}\right|<\sigma_{1} \frac{2\left\|\mathbf{h}_{1}\right\|^{2}}{\sqrt{10}}\right)\right)}\right. \\
\left|x_{1, I}\right|=\sigma_{1} \frac{1}{\sqrt{10}}\left(2+(-1)^{\left.I\left(\left|p_{12, R} x_{2, I}+p_{12, I} x_{2, R^{-}} y_{1, I}\right|<\sigma_{1} \frac{2\left\|\mathbf{h}_{1}\right\|^{2}}{\sqrt{10}}\right)\right)}\right.
\end{array}
$$

Fig. 5. $2 \times 2$ BICM system. Frame length of lower rate stream is fixed to

For QAM64-QPSK case bit metrics will be the same as those for QAM16-QPSK. For the case of QAM64-QAM16, bit metric for QAM16 will be (21) with the values of $x_{2, R}$ and $x_{2, I}$ given by (16). Using similar techniques, bit metrics for other combinations of higher order constellations can be found.

Fig. 5 shows the performance of proposed simplified metrics in a $2 \times 2$ BICM MIMO OFDM using rate- $1 / 2$ punctured turbo code proposed for 3GPP LTE [5]. The MIMO channel has iid Gaussian matrix entries with unit variance and the channel is independently generated for each time instant.

\section{Conclusions}

In this paper we have proposed low complexity MF based bit metrics for BICM SISO and BICM MIMO OFDM systems. In the case of BICM SISO systems, the proposed metrics are merely scaled versions of MF outputs and involve no distance computations and comparisons. Whereas in the case of MIMO systems, the proposed metrics reduce one complex dimension of the system. These low complexity bit metrics being based on MF outputs and devoid of any division operation can easily be implemented in the receiver structures for forthcoming wireless systems taking into account the existing hardware limitations.

\section{ACKNOWLEDGMENTS}

Eurecom's research is partially supported by its industrial partners: BMW, Bouygues Telecom, Cisco Systems, France Télécom, Hitachi Europe, SFR, Sharp, ST Microelectronics, Swisscom, Thales. The research work leading to this paper 1296 information bits.

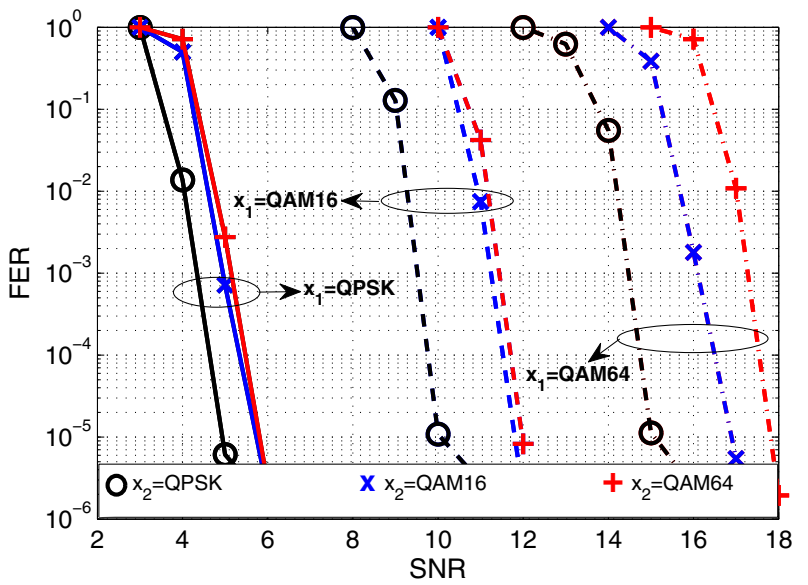

has also been partially supported by the European Commission under SAMURAI and IST FP7 research network of excellence NEWCOM++.

\section{REFERENCES}

[1] E. Zehavi, "8-PSK trellis codes on rayleigh channel," IEEE Transactions on Communications, vol. 40, pp. 873-884, May 1992.

[2] G. Caire, G. Taricco, and E. Biglieri, "Bit-interleaved coded modulation," IEEE Transactions on Information Theory, vol. 44, no. 3, pp. 927-946, May 1998.

[3] 802.11n, Enhancements for Higher Throughput. IEEE 802.11 WG. IEEE 802.11n/D1.0 Draft Amendment, 2006.

[4] $802.16 \mathrm{~m}$, Draft IEEE $802.16 \mathrm{~m}$ Evaluation Methodology. IEEE 802.16m-07/037r1, 2007.

[5] LTE, Requirements for Evolved UTRA (E-UTRA) and Evolved UTRAN (E-UTRAN). 3GPP TR 25.913 v.7.3.0, 2006.

[6] F. Tosato and P. Bisaglia, "Simplified soft-output demapper for binary interleaved COFDM with application to HIPERLAN/2," in IEEE International Conference on Communications, ICC 2002., vol. 2, 2002, pp. 664-668 vol.2.

[7] E. Akay and E. Ayanoglu, "Low complexity decoding of bit-interleaved coded modulation for M-ary QAM," in IEEE International Conference on Communications, ICC 2004, vol. 2, June 2004, pp. 901-905.

[8] B. Hochwald and S. t. Brink, "Achieving near-capacity on a multipleantenna channel," IEEE Transactions on Communications, vol. 51, pp. 389-399, Mar. 2003.

[9] F. Kaltenberger, R. Ghaffar, R. Knopp, H. Anouar, and C. Bonnet, "Openairinterfacean experimental platform for next generation wireless networks," URASIP Journal on Communications and Networking, 2010.

[10] G. J. Foschini and M. J. Gans, "On limits of wireless communication in a fading environment when using multiple antennas," Wireless Personal Communications., vol. 6, no. 3, pp. 311-335, March 1998. 